\title{
Road Extraction Using an Improved Snake Model and CART
}

\author{
Yi-Nan Lu, Zhe Zhang, Xiao-Ni Liu, Yun-Fan Du \\ College of Computer Science and Technology, \\ Jilin University, \\ Changchun, China \\ E-mail: zhangzhe14@mails.jlu.edu.cn,yn@jlu.edu.cn
}

\begin{abstract}
Road Extraction from remote sensing images has been an important research topic. It is difficult to extract the road quickly and reliably due to the complexity of the road features. In this paper, an improved GVF-Snake algorithm as a segmentation method automatically labels training samples to reduce the complexity of the manual labeling data, and a Classification and Regression Tree method is used to extract the roads from remote sensing images by classification. The experiments indicate that the proposed method can efficiently and automatically extract the roads from remote sensing images.
\end{abstract}

Keywords-road extraction; GVF-Snake; classification; CART

\section{INTRODUCTION}

Remote sensing (RS) technology plays an important role in agriculture, military, environment inspection, and resource survey. High resolution RS images can be used for the deep applications such as road extraction, water extraction and building extraction. It means that we can get more concrete road information from RS images [1, 2]. Road extraction methods are classified as classification-based, knowledgebased, mathematical morphology, active contour model and dynamic programming [3]. The features in a road image can be generally summarized as geometric features, texture features, and photometric features [4, 5]. Supervised classification methods as one type of classification methods need to select the features and train the labeled samples to obtain the classifiers. They mainly consist of support vector machine (SVM), artificial neural network (ANN), and Markov random fields (MRF).

Support vector machines are supervised learning models in machine learning. Song and Civco used a two-step SVM approach for classifying the images and detecting road networks [6]. In [7], a multiclass SVM method proposed by Simler accurately detected the roads from multispectral data. A neural network can be employed to effectively classify almost all the forms of the remote sensing data [8]. A robust $\mathrm{BP}$ algorithm and image processing techniques [9] were used for the road identification via classifying directly the edges and spectral information of the image pixels. The Markov random field is widely applied to edge detection, image segmentation, stereo matching and so on [10]. In [11], Zhu Daming established a MAP-MRF framework to segment the images and extract the roads. Further details of these methods can be found in [3].

Snake models have been widely used for image segmentation. Their main idea is summarized as follows: the original contours are continuously deformed under the common restriction of internal and external forces until the conditions are satisfied. Many snake models have been applied to the road extraction [12]. Due to the extracted contours highly dependent on the initialization of the snake model, Xu proposed a snake model based on gradient vector flow (GVF) to solve this problem [13], named as (GVFSnake).

Classification and regression tree (CART) was developed by Leo Breiman et al. [14]. CART uses the historical data to construct so-called decision tree. CART has been used for many tasks, for example, classifying images [15], predicting wave heights [16], determining sources of contamination [17], and studying the clinical epidemiology [18] and so on.

This paper mainly focuses on the algorithms of extracting roads automatically from images with high resolution. Improved GVF-Snake algorithm extracts positive samples as training samples. Then, CART learns from the training samples and produces a binary tree as the road extraction rules. Finally, by applying those rules and extracting the skeleton, we can get a road network.

Section II describes the improved GVF-Snake algorithm and CART algorithm, and the results are shown in Section III followed by the conclusion in Section IV.

\section{THE IMPROVED ALGORITHM AND CART}

In this paper, we use a supervised classification to extract the roads from RS images. The samples are labeled automatically using the improved GVF snake model. The decision tree is used to classify the images.

\section{A. Improved GVF-Snake Model}

A GVF-Snake model introduces GVF as an external force of the object edge to replace the external force of traditional Snake algorithm, and uses a diffusion equation to smooth. The goal of GVF is to search the minimum $w(x, y)=$ $[u(x, y), v(x, y)]$ in the following formula (diffusion equation).

$$
E=\iint \mu\left(u_{x}^{2}+u_{y}^{2}+v_{x}^{2}+v_{y}^{2}\right)+|\nabla f|^{2}|v-\nabla f|^{2} d x d y
$$

Where $f(x, y)$ is image function, $\nabla f$ is gradient field of $f$, $\mu$ is weight coefficient and proportional to image noise.

The road boundaries are generally symmetric and may be pressed into single contours when they don't look obvious. Based on the feature of the boundary, a new internal support function is introduced. The distance from a contour point to a road centerline is used as support value. As shown in Fig. 1, 
a right contour point may be pushed into $\mathrm{A}$ or $\mathrm{B}$ when the external force acts on it. Two cases are considered when the distance is less than the given threshold. It should be deleted if it is pushed from B into A. When a contour point is pushed into $\mathrm{B}$, a compensation value should be added to the contour point around the object edge during next iteration, as shown in Fig. 1.

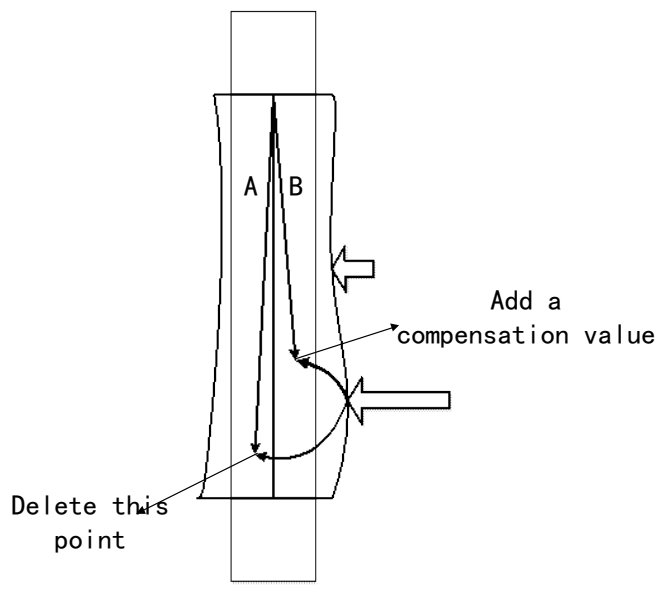

Figure 1. Both cases when contour points are converged.

A internal support function $\operatorname{dis}(x, y)$ is defined as the distance from a contour point $(x, y)$ to a road centerline and $\operatorname{vec}(x, y)$ is a container of the contour points and defined as:

$$
\begin{gathered}
\text { vec }(x, y)= \begin{cases}\operatorname{vec}(x, y) & \text { dis }(x, y)>=\mid \text { threshold } \mid \\
\operatorname{vec}(x, y)-(x, y)+\left(x^{\prime}, y^{\prime}\right) & \text { B region } \\
\operatorname{vec}(x, y)-(x, y) & \text { A region }\end{cases} \\
\qquad\left\{\begin{array}{l}
x=x+\mid \text { threshold }_{x} \mid \\
y=y+\mid \text { threshold }_{y} \mid
\end{array}\right.
\end{gathered}
$$

Where threshold $=\left(\right.$ threshold $_{x}$, threshold $\left._{y}\right), \mid$ threshold $\mid$ is half of the width of a road.

\section{B. CART Algorithm}

CART as a supervised classification method, uses the training samples to construct a binary tree. CART defines a measure to select an appropriate splitter in the tree. A leaf node contains some samples, which is expected to belong to the same category.

CART algorithm uses the Gini index as a criterion to measure the purity of data. The value of Gini is lower and the purity of the samples is higher. Gini index can be calculated as follows:

$$
\operatorname{Gini}(D)=1-\sum_{K=1}^{K} \frac{\left|C_{k}\right|^{2}}{|D|}
$$

Where D is a sample set; $\mathrm{C}_{k}$ is a sample subset belonging to Type $k$ of $\mathrm{D} ; \mathrm{K}$ is the number of categories. When we calculate all the GINI indexes of a splitting value for an attribute, and get the Gini Split Info (i.e. GiniGain), which is used to select the desirable attribute for each code in a tree.

$$
\operatorname{Gini}_{\text {split }}(D)=\sum \frac{N_{i}}{\mathrm{~N}} \operatorname{Gini}\left(D_{i}\right)
$$

Where $N$ is the number of training samples; $N_{i}$ is the number of $i$-th subset.

For CART, $i=1$, 2, we compute the GiniGain as follows:

$$
\operatorname{Gini}_{\text {split }}(D)=\frac{N_{1}}{N} \operatorname{Gini}\left(D_{1}\right)+\frac{N_{2}}{N} \operatorname{Gini}\left(D_{2}\right)
$$

When we get a sufficient number of the samples, we can use these sample data to train a decision tree. The training process mainly selects the splitter based on the minimal GiniGain to build a binary tree. This algorithm stops when the samples belong to the same class in nodes or the decision tree height reaches the threshold value set by the user. Finally we can get the whole classification rules used for classifying the new RS image.

\section{EXPERIMENTS}

Two algorithms are used to segment the fuzzy edge roads and label the pixels in the image, one is original GVF-Snake and the other is our improved GVF-Snake. The results of original GVF-Snake algorithms in segmenting the given image after two time iterations are shown in Fig. 2(a) and (b). Fig. 2(c) and 2(d) shows the results of improved GVF-Snake algorithm after two time iterations. Fig. 2(b) shows that contour has been pushed into a line, but Fig. 2(d) shows that contour line converges to the object edge perfectly. The results of the experiment show that the improved algorithm has better performance in segmentation than the original algorithm after two iterations.

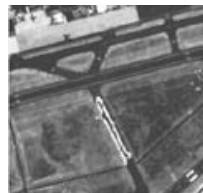

(a)

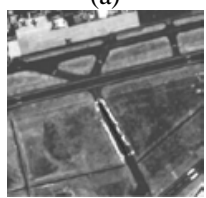

(c)

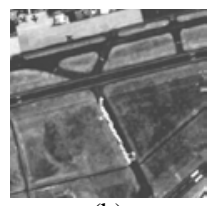

(b)

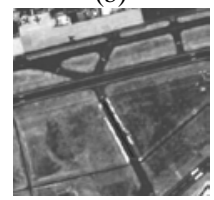

(d)
Figure 2. The experimental results of improved and original algorithms. (a) First iteration result of GVF-Snake (b) Second iteration of GVF-Snake (c) First iteration result of improved GVF-Snake (d) Second iteration result of improved GVF-Snake.

Fig. 3 shows that the more the algorithm runs, the more number of the contour points will be gotten. 


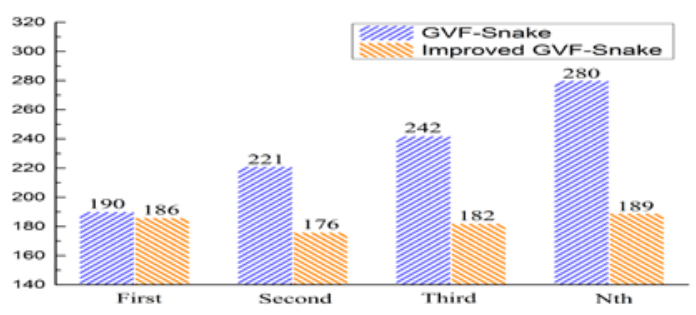

Figure 3. The comparison of improved and original algorithm on contour points.

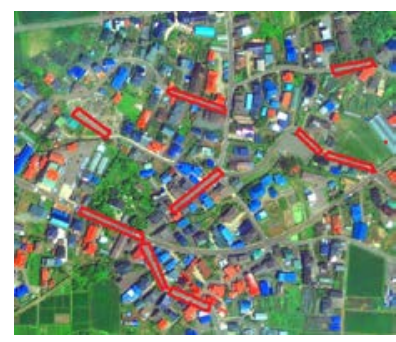

(a)

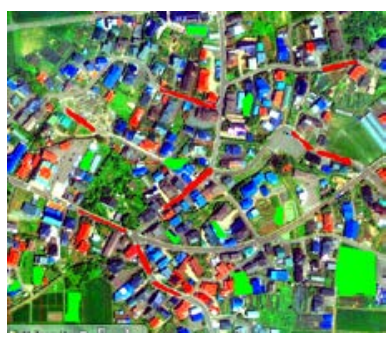

(b)
Figure 4. The image with the initial road outline and the image colored with the training samples.

In the experiment, Fig. 4(a) is the initial road outline image by using improved GVF-Snake. In Fig. 4(b), the red areas show that positive samples are extracted by improved GVF-Snake, and the green areas show that negative samples are extracted by manual way. We have acquired sample information as shown in Table I.

TABLE I. NUMBER OF SAMPLES

\begin{tabular}{|c|c|c|}
\hline $\begin{array}{c}\text { Positive/negative } \\
\text { samples }\end{array}$ & Number of regions & Number of samples \\
\hline Positive samples & 9 & 32175 \\
\hline Negative samples & 11 & 37854 \\
\hline
\end{tabular}

Considering the complexity of the objects in a RS image, we should select several types of objects as negative samples. In this RS image, we select fields, buildings, shadow, and vegetation as negative samples.

In the experiment, a RS image has three bands: R band, $\mathrm{G}$ band and $\mathrm{B}$ band. So, we use three bands as three attributes for the feature classification. It is two-class classification problem. We get a binary tree using the above CART. This tree can extract the roads from the remote sensing image after images processing techniques including geometrical calibration and tiling, and the three attributes are represented by B1, B2, and B3 respectively in this tree. Through training of the positive and negative samples by CART, we get the rules shown in Fig. 5.

The next step is to apply these rules to each pixel of the image. If the band value of the pixel matches the rules, we mark this pixel as black color; else, we mark it as white color. The basic framework of the road has been extracted in the entire image according to the rules.
Here, we have obtained the overall outline of the main roads, as shown in Fig. 6. The main roads have been extracted entirely, and some slip roads also been extracted effectively. We compare with the results using the original snake, GVF-snake and our method in terms of integrity and accuracy, our method has achieved higher performance, as shown in Table II.

The final step is to extract the skeleton of the roads by using Maximal disc algorithm, as shown in Fig. 7 (a). Fig. 7 (b) is the original image jointing with the skeleton. The extraction effect is good.

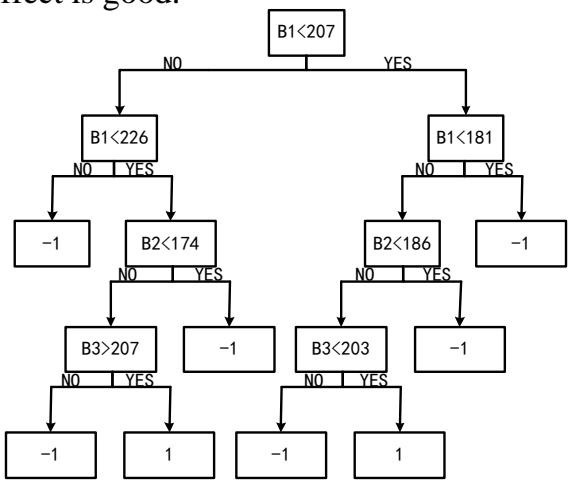

Figure 5. The binary tree generated by CART.

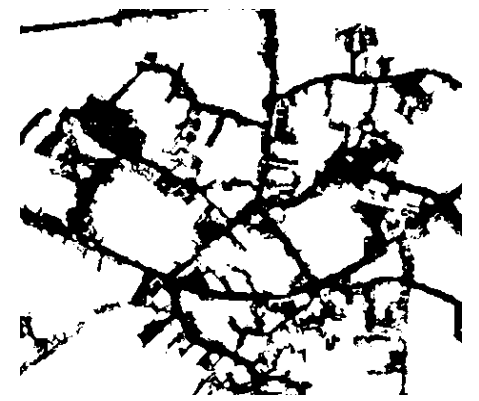

Figure 6. The result of extraction by CART.

TABLE II. THE RESULTS USING DIFFERENT METHODS.

\begin{tabular}{|c|c|c|}
\hline & Integrity & Accuracy \\
\hline Snake & $92 \%$ & $90.5 \%$ \\
\hline GVF-snake & $93.7 \%$ & $91 \%$ \\
\hline Our method & $94 \%$ & $92 \%$ \\
\hline
\end{tabular}

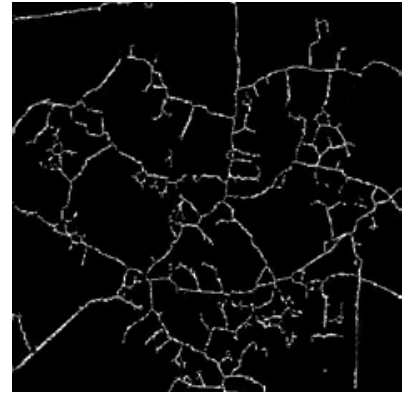

(a)

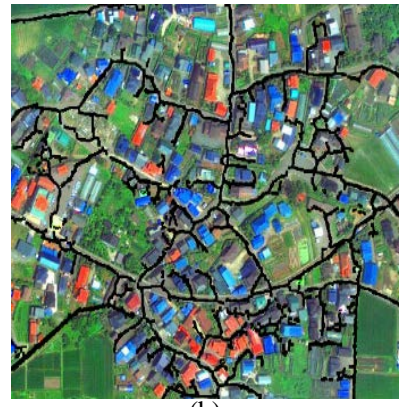

(b)
Figure 7. The results of skeleton extraction. 


\section{CONCLUSION}

In this paper, we propose an automatic algorithm to extract the roads from RS images based on improved GVFSnake and CART. The improved GVF-Snake as segmentation method can label the positive samples without manual work, which are further used for training the CART classifier. We train CART to get the classification rules and finally get the road network. Compared to the original snake and the GVF snake, our method has a higher accuracy and performance. We will select the different types of features for CART algorithm in the future work.

\section{ACKNOWLEDGMENT}

Supported by Specialized Research Fund for the Doctoral Program of Higher Education, China（No. 2013006111005 4)

\section{REFERENCES}

[1] Mena, J.B. and Malpica, J.A. "An automatic method for road extraction in rural and semi-urban areas starting from high resolution satellite imagery,” Pattern Recognition Lett. 26(9), 2005, pp.12011220.

[2] Dal Poz A P, Do Vale G M. "Dynamic programming approach for semi-automated road extraction from medium-and high-resolution images," ISPrS Archives, 2003, 34(Part 3): W8.

[3] Wang, W., Yang, N., Zhang, Y., Wang, F., Cao, T., \& Eklund, P. “A review of road extraction from remote sensing images," Journal of Traffic \& Transportation Engineering, 2016, 3(3), 271-282.

[4] Ma Li, Chen Jun. "Classification and application of context information in road extraction," Geomatics World, 2008, 6(4): 58-60.

[5] Zhang, Q. and Couloigner, I. "Automated road network extraction from high resolution multi-spectral imagery,” In: Proc. ASPRS Annual Conf., Reno, Nevada, 2006.

[6] M. J. Song and D. Civco, "Road extraction using SVM and image segmentation,” Photogramm. Eng. Remote Sens., vol. 70, no. 12, 2004, pp. 1365-1371.
[7] Simler, C. "An improved road and building detector on VHR Images," In: International Geoscience and Remote Sensing Symposium, Vancouver, 2011.

[8] Mas J F, Flores J J. "The application of artificial neural networks to the analysis of remotely sensed data," International Journal of Remote Sensing, 2008, 29(3):617-663.

[9] Kuo-Tu K. "A Hybrid Road Identification System Using Image Processing Techniques and Back Propagation Neural Networks," Mississippi State University, 1995.

[10] Yousif, O., and Y. Ban. "Improving SAR-Based Urban Change Detection by Combining MAP-MRF Classifier and Nonlocal Means Similarity Weights," IEEE Journal of Selected Topics in Applied Earth Observations \& Remote Sensing , 2014:4288-4300.

[11] Da-Ming Zhu, Xiang Wen and Chun-Li Ling "Road Extraction Based on the Algorithms of MRF and Hybrid Model of SVM and FCM," 2011 International Symposium on Image and Data Fusion.

[12] Yang, Hu, and K. J. Zu. "Road Extraction from Remote Sensing Imagery Based on Road Tracking and Ribbon Snake,” Pacific-Asia Conference on Knowledge Engineering and Software Engineering IEEE Computer Society, 2009:201-204.

[13] Xu C, Prince J L. "Snakes, shapes, and gradient vector flow,” IEEE Transactions on Image Processing, 1998, 7(3): 359-369

[14] Breiman, and Leo. "Classification and regression trees,” Wadsworth International Group, 1984.

[15] Li, Hui, J. Sun, and J. Wu. "Predicting business failure using classification and regression tree: An empirical comparison with popular classical statistical methods and top classification mining methods,” Expert Systems with Applications An International Journal 37(8), 2010:5895-5904.

[16] Mahjoobi, J., and A. Etemad-Shahidi. “An alternative approach for the prediction of significant wave heights based on classification and regression trees,” Applied Ocean Research 30(3), 2008:172-177.

[17] Jan Gregor, Nick Garrett, Brent Gilpin, Cliff Randall, \& Darren Saunders. "Use of classification and regression tree (cart) analysis with chemical faecal indicators to determine sources of contamination," New Zealand Journal of Marine \& Freshwater Research, 36(2), 2008:387-398.

[18] Marshall, Roger J. "The use of classification and regression trees in clinical epidemiology,” Journal of Clinical Epidemiology 54(6), 2001: 603-609. 